\title{
A Brief Discussion on the Building of a Harmonious Medical Environment with the Relationship between Doctors and Patients Ning-Can LONG
}

\author{
The First Affiliated Hospotal ,Jinan University,Guangzhou
}

1413615985@qq.com

Key words: Doctor-patient relationship, Harmonious medical treatment.

\begin{abstract}
In recent years, with the rapid development of China's market economy, the relationship between doctors and patients is becoming increasingly intense, and the resulting medical disputes are mushrooming. Facing the increasingly prominent doctor-patient relationship, our medical workers should actively seek the source, explore countermeasures, improve the quality of medical care, understand relevant legal knowledge, strengthen communication between doctors and patients, reduce the occurrence of medical disputes, and build a harmonious doctor-patient environment.
\end{abstract}

\section{Introduction}

With the continuous development of social market economy, our demand for health becomes more and more intensive, frequent medical activities also tends to be obvious, but also to let everyone get to the medical staff is far higher than other industries have different interests and misunderstanding; agree that patients pay check the high cost of treatment should obtain satisfactory curative effect and medical service to the hospital; exaggerated "supreme interests of patients". When there is a huge gap between patients' and their families' actual satisfaction and expectations and reality, they will immediately feel their interests have been seriously damaged, and the inevitable doctor-patient will start. Intense doctor-patient relationship not only affects the physical and mental health of patients and their families, but also interferes with the normal order of diagnosis and treatment in medical institutions. What's more, the trust between people and people is greatly reduced. How to correctly handle the relationship between doctors and patients has become a serious problem for medical staff. Through the investigation and analysis of medical patients in medical disputes, the aim is to build a harmonious medical environment.

\section{Doctor-Patient Relationship}

There are two kinds of relationship between doctor and patient in narrow sense and broad sense. The narrow relationship between doctors and patients refers to the interaction and connection between patients themselves and medical staff. "Medical" refers to medical institutions and their medical staff, while medical staff refer to all kinds of doctors, nurses and medical administrators. Under normal circumstances, medical staff's negligence, misoperation and inappropriate attitude are the main causes leading to doctor-patient disputes. The cognitive bias of patients is another major cause of conflicts between doctors and patients. "Suffering" refers to the physical or mental illness, the need for professional treatment to regain the health of the patient.

\section{Analysis of Problems in Hospital Doctor-Patient Relationship Management System}

\section{The Awareness of Prevention Needs to be Strengthened}

We should strengthen the field exercise, pay attention to safety education, when the doctor-patient conflicts occur, you can calmly and smoothly resolve; but if you usually do not pay attention to the cultivation of awareness of the measures for crisis prevention and self-protection is not that, in the face of the doctor-patient contradiction, many medical personnel suffer great harm, or even lose life. In addition, because many medical staff can not predict the whole process of Doctor-patient 
Conflict, we cannot foresee the trend of physician-patient relationship, and we cannot treat the contradiction between doctors and patients scientifically.

As a result of the continuous improvement of hospital patient visits, all departments put the main energy on how to cure the patients. To ignore the unstable factors behind various medical establishments may exist in the doctor-patient relationship arising in advance of the doctor-patient conflicts may face reflection and anticipation, and lectured in psychology, and ready to work in manpower, material and financial resources, prevention awareness is weak, lack of sense of crisis judgement ability, when the real contradiction occurs, many medical personnel are luck, that may lead to devastating consequences for more than.

\section{A lack of Correct Judgment}

The whole hospital's hardware and software environment have not been well evaluated. From the aspect of hardware, it mainly includes all kinds of information equipment, emergency security equipment used by security and other related hardware. On the software aspect, it is mainly the assessment of the quality and ability of the related personnel. For example, is the quality of all medical staff able to meet the requirements of the post capacity? Which departments have a greater risk of a doctor-patient crisis? What is the consciousness of the medical staff? It is necessary for us to further strengthen the assessment of the related environment so that we can be further grasped so as to improve the ability to deal with it.

Building a harmonious atmosphere for medical treatment

\section{Improving the Consciousness of Prevention}

By establishing and improving the education and training system, we can strengthen the training of medical personnel and improve their awareness of prevention. First of all, improve the relevant system. Regular or irregular training of doctor-patient relationship knowledge and coping skills, learn how to resolve conflicts between doctors and patients, form a sense of the whole staff, and let them know what measures should be taken to prevent and protect themselves. Secondly, strengthen medical ethics education. The medical ethics education and training, and strengthen medical ethics of doctors, the doctor reminded to abstinence, not big, hard to carry out medical activities, from their start, from the source to avoid the conflicts between doctors and patients. Finally, regular or irregular training and assessment of related business knowledge are carried out.

\section{Information-Based Equipment for Upgrading}

Related equipment need to open and not become a kind of decoration, the medical process can be monitored at the same time with a special maintenance, equipment failure in time, so it is good for medical information collection, information processing, information processing and utilization, can provide evidence and information corresponding to the occurrence of medical accidents, the doctors and patients the contradictions are resolved timely and effective.

\section{Perfect Emergency Plan}

To improve the emergency plan is the key to cope with the contradiction between doctors and patients, and also to improve the efficiency of coping with the contradiction between doctors and patients.

Through the formation of a perfect crisis risk assessment plan, we should form positive coping strategies so as to transform the attitude towards risk from negative coping to positive coping, so as to enhance our ability to deal with risks.

\section{Take the Relevant Measures Decisively}

One is to improve the skills of dealing with things. We can make some publicity on the skills of dealing with the relationship between doctors and patients. When a more serious doctor-patient relationship took place, the family members demonstrated at the door of the hospital, expressing their dissatisfaction with the hospital by sitting quietly, pulling banners, setting off firecrackers, shouting and so on, and demanded that the hospital pay for the losses, or even further intense acts. 
The relevant medical staff should first stabilize the scene and take measures to avoid intensifying the contradictions between the two sides.

The two is to master the information quickly and understand the details of the event. Information is the basis for taking measures. Therefore, we can quickly inform the relevant people through the information above, so that we can understand the focus of the concerned parties and grasp the needs and pain points of the other party, so that we can take more targeted measures. For example, families that are caused by the negligence of the hospital, then according to the information about why the families do; or through information analysis, this is belongs to the occupation medical acts if occupation medical acts would be to take decisive measures to control the scene.

\section{Establish an Effective Communication Mechanism}

Communication is the key work to solve the problem. Both doctors and patients need to communicate information and reach consensus through communication and communication, so as to achieve the purpose of solving them. We can improve the communication skills of the people concerned. By means of training, simulation and testing, the related medical staff have some communication skills. Through training, simulation, testing and so on, help related personnel to learn the way of intonation and speech when communicating with crisis, so that they can learn to listen to the opinions of the patients, try to understand each other from the perspective of the patients, and weigh the other's views. A better understanding of the real cause of the whole thing and where to clear the crux of the problem. Better to provide the basis for the two sides to solve the problem together, and let the hospital better put forward the solution. In addition, by establishing a fast communication mechanism within the hospital, the relevant medical staff can get more information before communicating with the patient, and strengthen the timeliness of communication, so that the hospital can make more efforts in advance and improve the possibility of solving the problem. The second is to build a platform for both doctors and patients to communicate. To strengthen the medical personnel according to the law practice consciousness, in daily medical work, medical personnel should timely diagnosis, patients' condition, take medical measures, possible medical risks to communicate with patients and their families, timely answers to the patient counseling, to perform the obligation of informing patients is medical institutions and medical personnel the legal obligations of [1].

\section{Establishing and Perfecting The Related Evaluation System}

In a certain period of time to carry out a comprehensive assessment of it, to find out the existing problems, and summarize the experience of the case. According to the actual situation, the evaluation work is standardized, and experience and lessons are shared. We can accelerate the improvement and improvement, and then promote the scientific and standardized process of management.

\section{Improving the Ability to Guide Public Opinion}

By enhancing communication ability with media and other media, we should set up a fine style of rigorous, realistic, quick response, be good at contingency and dare to take responsibility, set up a keen sense of public opinion, guide media to report relevant facts, and avoid distorting facts as much as possible. In addition, we should respond in time and deal with it in time.

Under the guidance of "the socialist thought with Chinese characteristics in the new era", which was put forward by the general secretary of the general secretary at present; which was put forward by the general secretary of the general secretary at present; We should combine the hospital work characteristics and practice, to "serve the people" as the center, with "medical quality" concept, to "heal" as the criteria; establish long-term working mechanism as the core, strictly grasp the standard of medical behavior, strengthen the doctor-patient communication, strengthen medical ethics construction, the construction of harmonious doctor-patient unremittingly the relationship between the promoters; establish a good doctor-patient relationship, timely communication with patients, to meet the needs of patients on medical knowledge, timely understanding of the condition changes [2]; let the people seek to enjoy real Huimin service, together to create mutual respect, mutual 
understanding, warm and harmonious medical environment.

\section{References}

[1] Zhu Weiliang, Wang Qiang. Look at the focus and difficulty of the construction of harmonious hospital [J]. Journal of Nanjing Medical University from the medical ethics status: Social Science Edition, 2007,7 (4): 314.

[2] Liu Junrong. Causes of defensive medicine and its impact on the relationship between doctors and patients [J]. Chinese Hospital Management Journal, 2003,19 (8): 494. 